\title{
Ongoing rubella outbreak among adults in Tokyo, Japan, June 2012 to April 2013
}

\author{
Yoshiyuki Sugishita, ${ }^{a}$ Takuri Takahashi, ${ }^{a}$ Narumi Horib and Mitsuru Aboc \\ Correspondence to Yoshiyuki Sugishita (e-mail: yoshiyuk@gmail.com).
}

Introduction: A large rubella outbreak has been occurring in Tokyo, Japan since June 2012. Rubella vaccination, introduced in Japan in 1976, has targeted different age groups, resulting in a large proportion of the current population being unvaccinated.

Methods: Rubella cases reported in Tokyo from 2 January 2012 to 21 April 2013 were analysed. A clinical case had generalized maculopapular rash, fever and lymphadenopathy; a laboratory-confirmed case was a clinical case with a positive serology or polymerase chain reaction test for rubella. A descriptive analysis of cases by age, sex, vaccination history and other epidemiological information was conducted.

Results: A total of 2382 cases were reported from all areas of Tokyo. Three-quarters were male ( $n=1823 ; 76.5 \%)$; the highest number of cases occurred among males aged 35-39 years and females aged 20-24 years. About a third of males (27\%) and females (32\%) reported never receiving rubella vaccination, with $68 \%$ and $56 \%$, respectively, having an unknown vaccination status.

Discussion: This outbreak reflects the changing, yet incomplete, immunization policies for rubella in Japan that may increase the risk of congenital rubella syndrome (CRS). To suppress the outbreak of rubella and prevent CRS cases, we recommend vaccination for the entire susceptible population.

$\mathrm{R}$ ubella is usually a mild, rash-producing, febrile illness in children; however, infection in pregnant women, especially during the first trimester, can result in still births, fetal death or congenital defects known as congenital rubella syndrome (CRS). Rubella vaccination was added to the Japanese national immunization schedule in 1976 and up until 1994 was limited to girls in grades seven to nine (ages 12-15). In 1995 vaccination of all children (12-90 months old) was introduced. According to the nationwide sentinel surveillance system, before 1998 there were an estimated 170000 or more rubella cases every year; ${ }^{1}$ since 1999 cases have decreased by one-quarter to onetwentieth. $^{2}$ A second dose of the measles-rubella (MR) vaccine was introduced in 2006 on entry to grade one (five to six years old). In 2011, administrative MR vaccine coverage was $95.3 \%$ at age one year and $92.8 \%$ at age five to six years. ${ }^{3}$ After a large measles outbreak in 2007 and establishing a goal of measles elimination by 2012, a catch-up programme using the bivalent vaccine was offered for grades seven and 12 (ages 12-13 and 17-18) from April 2008 through March 2013.
As a consequence of these vaccination policies, different age cohorts have different levels of protection against rubella. In the 2012 annual national seroepidemiologic survey, $73 \%-86 \%$ of males and $97 \%-98 \%$ of females aged $30-50$ years were seropositive for rubella antibody, while $90 \%$ or more of children aged over one year and adolescents of both sexes were seropositive. ${ }^{4}$

Case-based surveillance for CRS started in 1999 in Japan; all physicians were required to report all CRS cases. During the period 1999-2011 there were 19 CRS cases reported in Japan, including three in Tokyo. In 2008, rubella surveillance in Japan changed from being part of the sentinel surveillance system, where a proportion of physicians reported cases, to being a disease notifiable by all health care providers. From 2008 to 2011, fewer than 50 rubella cases were reported per year in Tokyo.

Since June 2012, after seven years of low incidence, a large increase in rubella notifications was

\footnotetext{
Epidemiological Information Section, Tokyo Metropolitan Institute of Public Health, Tokyo, Japan.

Disease Control and Prevention Center, National Center for Global Health and Medicine, Tokyo, Japan.

Ogasawara Branch Office, Tokyo Metropolitan Islands Public Health Center, Tokyo, Japan.

Submitted: 20 May 2013; Published: 1 August 2013

doi: 10.5365/wpsar.2013.4.2.011
} 
Figure 1. Number of reported rubella cases and proportion of males by epidemiological week of diagnosis, Tokyo, Japan, Week 1, 2012-Week 16, 2013

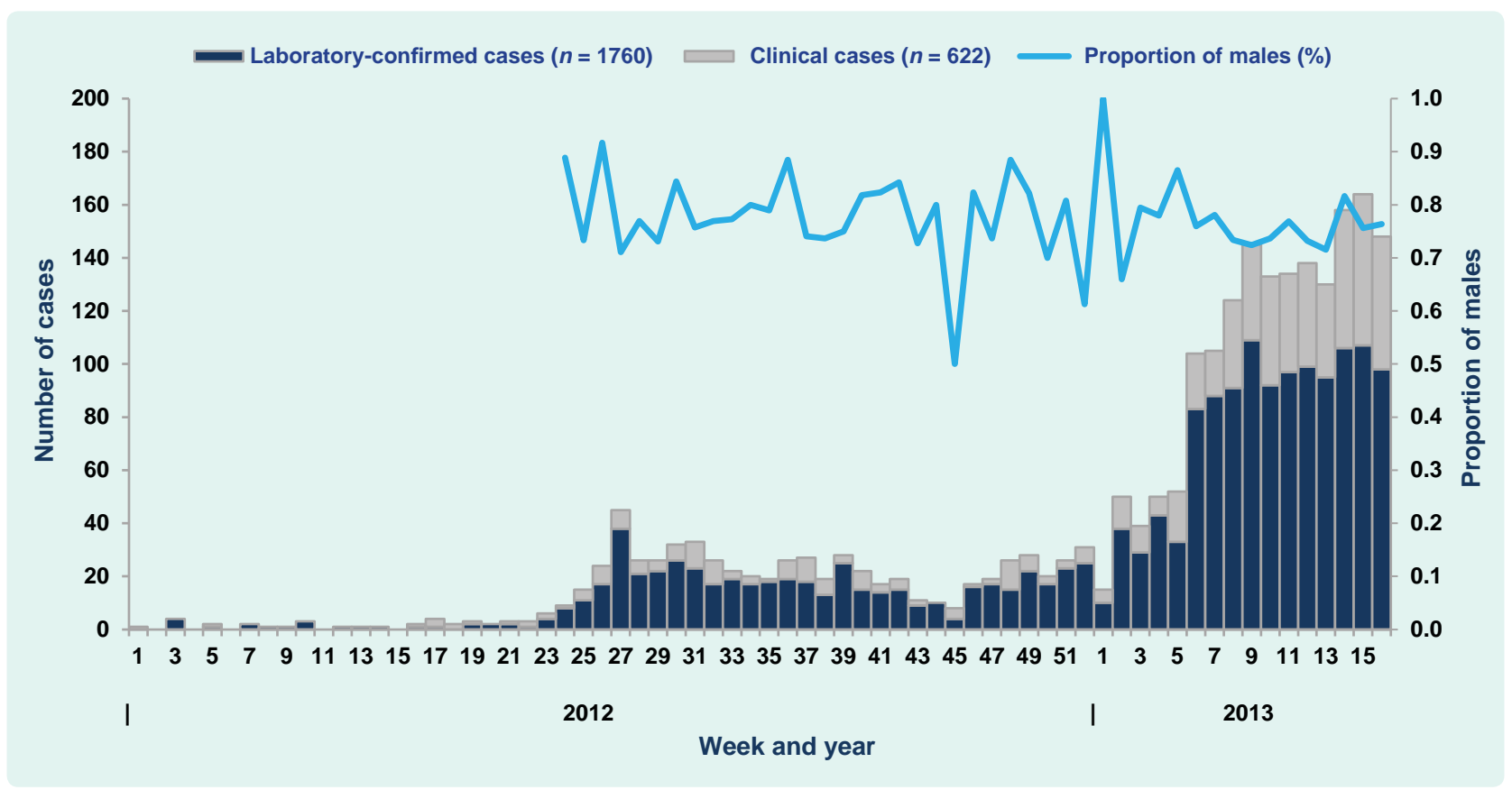

observed in Tokyo. Here we describe the epidemiology of rubella cases notified in Tokyo from January 2012 to April 2013.

\section{METHODS}

Rubella cases with a diagnosis date between 2 January 2012 and 21 April 2013 in Tokyo were extracted from the National Epidemiological Surveillance of Infectious Diseases (NESID) system on 1 May 2013. NESID is the nationwide case-based surveillance system; rubella was added in January 2008. All physicians are required to report all clinically diagnosed and laboratoryconfirmed rubella cases to local health officials through a designated form. Case details, which can be accessed at the national level, are then entered into the centralized notification system by local health officials. Case details include diagnosis method (clinical or laboratory), age, sex, diagnosis date, suspected route and location of transmission, vaccination history, complications and location of medical facility. For Tokyo, this surveillance system covers approximately 13 million people, 31 public health centres and approximately 12000 medical facilities.

A clinical rubella case was defined as a person with generalized maculopapular rash, fever and lymphadenopathy. A laboratory-confirmed case was a clinical case with detection of rubella through polymerase chain reaction (PCR), rubella-specific IgM antibody or seroconversion tests. As the weekly number of reported rubella cases in Tokyo was between zero and four during 2008 and 2011, a rubella outbreak was defined as the continual occurrence of more than four rubella cases in a week.

History of vaccination was based on either maternity health records or patient recall. Gender- and agespecific notification rates per 1 million inhabitants were calculated using the October 2012 census estimates for Tokyo as denominator.

\section{RESULTS}

A total of 2382 rubella cases (18 per 100000 population) were reported between 2 January 2012 (week 1) and 21 April 2013 (week 16) from 917 hospitals and clinics throughout Tokyo. From week six in February 2013, more than 100 cases were notified per week. As of 1 May 2013 (week 18), total cases for 2013 were 1689-89 times to higher than the same period of 2012 (Figure 1).

Most cases ( $n=1760 ; 73.9 \%$ ) were laboratory confirmed; 242 by PCR. There were 1823 (76.5\%) male cases and 18 pregnant women cases. The overall 
Figure 2. Number and rate of reported rubella cases by sex, age group and vaccination status, Tokyo, Japan, Week 1, 2012-Week 16, 2013
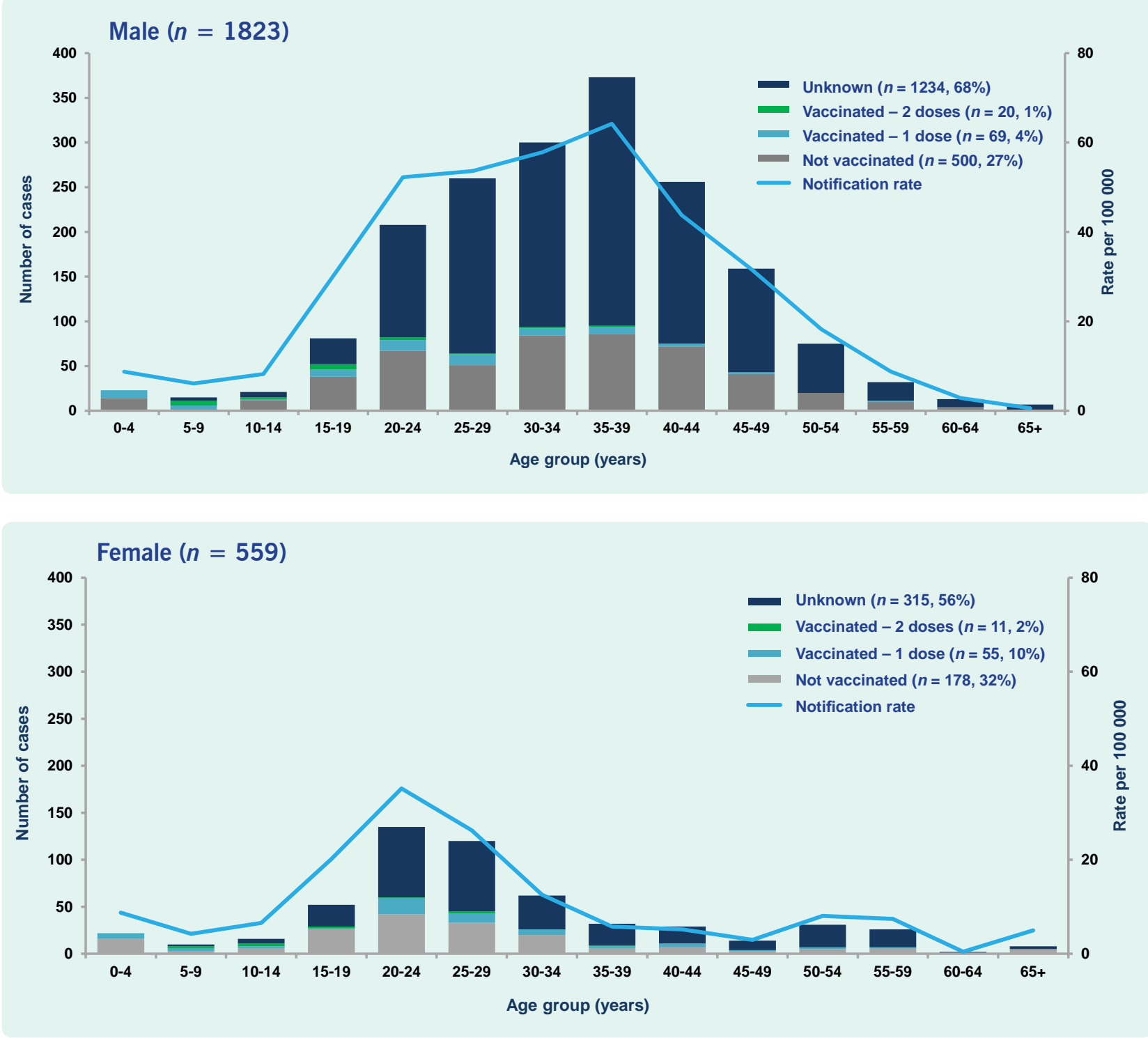

male-to-female ratio was $3.3: 1$; in 2012 it was $3.6: 1$, whereas in 2013 it was 3.1:1. The median age of notified cases was 35 years for males and 26 years for females (Figure 2). Males aged 30-39 years were the most commonly notified age group, although in December 2012, there was an increase in notifications for females aged 20-29 who had not been vaccinated. In 2013, notifications for children aged less than 15 years and adults aged more than 50 years emerged. Almost a third of males (27\%) and females (32\%) reported never receiving a rubella vaccination with $68 \%$ and $56 \%$, respectively, having an unknown vaccination status. Complications included thrombocytopenic purpura $(n=9)$, hepatic dysfunction $(n=7)$, encephalitis $(n=5)$ and meningitis $(n=1)$.
Japan was the reported place of exposure for 2366 cases, 1635 of which were in Tokyo. Nine cases reported being exposed outside Japan; exposure location for seven cases was unknown. Further exposure information was reported for 501 (21\%) cases and included the workplace $(n=200)$, family or housemates $(n=113)$, crowded places $(n=92)$; friends $(n=36)$, welfare facilities $(n=28)$, schools $(n=25)$ and nurseries $(n=7)$.

Outbreaks in companies, schools or institutions were also reported $(n=17)$ with the index cases all being adults. There was also secondary and tertiary transmission of rubella among unvaccinated people in most of these places. 


\section{DISCUSSION}

The current rubella outbreak in Tokyo is part of an ongoing larger outbreak of rubella across Japan, with more than $60 \%$ of cases reported nationally being from Tokyo and surrounding areas. ${ }^{3}$ Most cases were reported in males aged 20-44 years, similar to the resurgence of rubella in Greece in $1993^{5}$ and the current outbreak in Poland. ${ }^{6}$ Potential reasons for this outbreak may include vaccine failure, high population density, lack of awareness in the population, insufficient isolation after disease onset and transmission from asymptomatic cases. We believe that this outbreak was strongly influenced by the history of selective immunization policies in Japan that left a large susceptible population. Poland also reported a similar history of selective immunization. ${ }^{6}$ In contrast, although Finland first provided rubella vaccination for school-age girls only, they have since achieved high coverage rates and rubella elimination. ${ }^{7}$

The current Japanese immunization schedule does not always provide free access to rubella vaccine or catch-up vaccination for adults and therefore comes with a financial burden. In March 2013 as an outbreak response, the Tokyo metropolitan government provided financial support to the 62 local governments for adult MR vaccination. All started offering free or reduced cost vaccine programmes; however, instead of vaccinating the susceptible population identified by this outbreak, i.e. adult males, most local governments targeted females of childbearing age and their partners. While the intent is to curtail the incidence of CRS, this effort may not be sufficient to suppress the current outbreak. ${ }^{8,9}$

Several countries have alerted the public, especially travellers, about the outbreak in Japan. ${ }^{10-13}$ We strongly recommend that travellers confirm their vaccination history before visiting Tokyo and that women in the early phases of pregnancy avoid visiting Tokyo or at least avoid crowds. The latter is an official recommendation by Ministry of Health, Labour and Welfare.

Not all cases in this outbreak were laboratory confirmed, with a quarter based on clinical symptoms only. These cases could have been many other diseases misclassified as rubella. Since more than half of vaccination histories were unknown, there may have been recall bias about vaccination histories. We were also unable to conduct further tests to determine the genotype of this outbreak. However, the current dominant genotypes in Japan were genotypes $1 \mathrm{E}$ and 2B. ${ }^{4}$ Kanagawa prefecture, located south of Tokyo, also reported that the circulating rubella virus was type $2 \mathrm{~B} .{ }^{14}$

In Tokyo, the incidence of rubella in 2012 was 52 cases per million population and in 2013 is 128 cases per million. To control rubella and prevent CRS, the WHO Regional Office for the Western Pacific set a target for rubella of less than 10 cases per million population by $2015 .{ }^{15}$ An increase of cases in pregnant women and three cases of CRS were notified in Tokyo during this outbreak, suggesting that Japan still has a way to go to reach this goal.

\section{Conflict of interest}

None declared.

\section{Funding}

None.

\section{Acknowledgements}

We acknowledge all public health staff, all notifying physicians in Tokyo and the surveillance officer from Tokyo metropolitan infectious disease surveillance centre for their contributions to this surveillance system. In addition, we acknowledge $\mathrm{Dr}$ John Kobayashi for specialized advice.

\section{References:}

1. National Institute of Infectious Diseases and Tuberculosis and Infectious Diseases Control Division, Ministry of Health, Labour and Welfare, Japan. Rubella, Japan, 1995-1999. Infectious Agents Surveillance Report, 2000, 21(1) (http://idsc.nih.go.jp/ iasr/21/239/tpc239.html, accessed 17 May 2013).

2. National Institute of Infectious Diseases and Tuberculosis and Infectious Diseases Control Division, Ministry of Health Labour and Welfare, Japan. Measles and rubella in Japan, as of March 2006. Infectious Agents Surveillance Report, 2006, 27(4):85-86 (http://idsc.nih.go.jp/iasr/27/314/tpc314.html, accessed 17 May 2013).

3. Tanaka-Taya $\mathrm{K}$ et al.; Centers for Disease Control and Prevention (CDC). Nationwide rubella epidemic - Japan, 2013. Morbidity and Mortality Weekly Report, 2013, 62:457-462. pmid:23760185

4. National Institute of Infectious Diseases and Tuberculosis and Infectious Diseases Control Division, Ministry of Health, Labour and Welfare, Japan. Rubella and congenital rubella syndrome in Japan, as of March 2013. Infectious Agents Surveillance Report, 2013, 34(4):87-89 (http://www.nih.go.jp/niid/en/iasr-e/865iasr/3469-tpc398.html, accessed 17 May 2013). 
5. Panagiotopoulos T et al. Increase in congenital rubella occurrence after immunisation in Greece: retrospective survey and systematic review. BMJ (Clinical Research Ed.), 1999, 319:1462-1467. doi:10.1136/bmj.319.7223.1462 pmid:10582926

6. Paradowska-Stankiewicz I et al. Ongoing outbreak of rubella among young male adults in Poland: increased risk of congenital rubella infections. Euro Surveillance: European Communicable Disease Bulletin, 2013, 18(21): pii: 20485 (http://www. eurosurveillance.org/ViewArticle. aspx?Articleld=20485, accessed 27 June 2013).

7. Davidkin I, Peltola H, Leinikki P. Epidemiology of rubella in Finland. Euro Surveillance: Communicable Disease Bulletin, 2004 9(4):pii=459 (http://www.eurosurveillance.org/ViewArticle. aspx?Articleld $=459$, accessed 27 June 2013).

8. World Health Organization. Rubella vaccines: WHO position paper. Weekly Epidemiological Record, 2011, 86(29):301316 (http://www.who.int/wer/2011/wer8629.pdf, accessed 17 May 2013).

9. Centers for Disease Control and Prevention (CDC). Elimination of rubella and congenital rubella syndrome-United States, 19692004. Morbidity and Mortality Weekly Report, 2005, 54:279282. pmid: 15788995

10. Taiwan CDC stresses importance of timely childhood vaccination and advises infants below one year of age and pregnant women having little or no immunity to rubella to avoid visiting endemic areas as this year's first imported rubella case confirmed.
Taiwan, Centers for Disease Control, 2013 (http://www.cdc.gov. tw/english/info.aspx?treeid=EEOA2987CFBA3222\&nowtreeid =D3C5BBCF8E60CF3D\&tid=FCD7B3F0F553A70F, accessed 17 May 2013).

11. Department of Public Health. Rubella Outbreak in Japan. Guam, Department of Public Health and Social Services, 2013 (http:// www.dphss.guam.gov/article/2013/04/11/rubella-outbreakjapan, accessed 17 May 2013).

12. Centers for Diseases Control and Prevention (CDC). Rubella (German measles) in Japan. Atlanta, Centers for Disease Control and Prevention, 2013 (http://wwwnc.cdc.gov/travel/notices/alert/ rubella-japan, accessed 27 June 2013

13. Public Health Agency of Canada. Rubella (German measles) in Japan and Poland. Ontario, Public Health Agency of Canada, 2013 (http://www.phac-aspc.gc.ca/tmp-pmv/notices-avis/noticesavis-eng.php? $i d=115$, accessed 27 June 2013).

14. National Institute of Infectious Diseases and Tuberculosis and Infectious Diseases Control Division. Flash Report of rubella virus in Japan. Tokyo, Ministry of Health, Labour and Welfare, 2013 (http://www.nih.go.jp/niid/en/iasr-rubella-e.html, accessed 27 June 2013).

15. Fact sheet on rubella and congenital rubella syndrome (CRS). Manila, World Health Organization Regional Office for the Western Pacific, 2012 (http://www.wpro.who.int/mediacentre/factsheets/ fs_20120228/en/index.html, accessed 27 June 2013). 